\title{
Evaluación de la producción científica de las revistas de ciencia de la información sobre el tema estudios métricos en SciELO
}

\author{
Evaluation of the scientific production from Scielo periodicals, in the area of information science,
} about the subject metric studies

\section{Ely Francina Tannuri de OliveIRA (1) y João Batista Ernesto de MORAES (2)}

(1) Universidade Estadual Paulista - UNESP - Avenida Hygino Muzzi Filho, 737 - CEP: 17525-900, Marília, São Paulo, Brasil. etannuri@flash.tv.br (2) jota@marilia.unesp.br

\begin{abstract}
Resumen
Se evalúa la producción científica de periódicos pertenecientes a la base SciELO en el área de Ciencia de la Información en lo que se refiere al tema estudios métricos en un periodo de dos años. Los objetivos específicos fueron identificar y analizar las tendencias presentes en la comunidad científica, con relación a esa temática, en las siguientes variables: subtemas específicos abordados, instituciones de origen, coautorías, periódicos más citados, país de origen y lengua, con la finalidad de caracterizar y subsidiar la visión actual de tendencias de investigación. Los temas más frecuentes son la producción científica y las cuestiones relacionadas a la evaluación de periódicos, evaluación de la producción científica a través de indicadores métricos, tales como, factor de impacto y análisis de citación, con el $48 \%$ de los artículos. Les siguen webometría, links, evaluaciones informétricas, cientométricas y bibliométricas también son contemplados, así como métodos cuantitativos en general, estos en menor porcentual. Los artículos sobre cuestiones más candentes (análisis de dominio y redes de comunicación científica) escasean. La Universidade de São Paulo, y su sistema de bibliotecas, es la más productiva con relación al tema $(20 \%)$; le siguen universidades extranjeras de España, México, EEUU, Uruguay, Bélgica y Francia $(17,77 \%)$, lo que demuestra el carácter internacional de la base SciELO y la diseminación del tema; además de una productividad con gran dispersión por los diferentes Estados de Brasil. Se resalta la importancia de las evaluaciones de la producción científica para visibilidad de la ciencia, que apunten los rumbos tomados en el ámbito nacional e internacional, suministrando un panorama de las instituciones y grupos de investigación más productivos dentro de esa temática. Se recomienda que estas evaluaciones sean extendidas a otros temas, bases de datos o bibliotecas electrónicas.
\end{abstract}

Palabrasclave: Estudios métricos. Evaluación de la producción científica. Bibliometría. Evaluación de periódicos. Redes de comunicación.

\begin{abstract}
The scientific production of periodicals that belong to the SciELO database in the area of Information Science specifically to the subject metric studies is evaluated in a two-years period. The existing trends in the scientific community, related to this thematic, have been analyzed in the following variables: specific subsubjects approached, institutions of origin, co-authors, more cited periodicals, native country and language, with the purpose of characterizing and subsidizing the current vision of research trends in this subject. The most frequent subjects are the scientific production and the questions connected to the evaluation of periodicals, evaluation of the scientific production through metric indicators, such as, impact factor and citation analysis, totalizing $48 \%$ of articles; followed by webometric, link, informetric, scientometric and bibliometric evaluations; and, finally, quantitative methods in general, these in minor percentage. The hotter questions in the subject, such as domain analysis and scientific communication nets, are seldom contemplated. The most productive institution is the Universidade de São Paulo, including its system of libraries $(20 \%)$, followed by foreign universities from Spain, Mexico, United States, Uruguay, Belgium and France $(17,77 \%)$, (demonstrating the international character of the SciELO base and the dissemination of the subject), and, later, the universities of the different States of Brazil with great dispersion. The analysis of others variables is still in progress. In conclusion, we point out the importance of the evaluations of scientific production for the visibility of the science, because it points the routes taken in the national and international scope, supplies a panorama of the most productive institutions and research groups inside this thematic, and provides a panoramic view of the Information Science area, that is having more and more relevance. We recommend extending the evaluation be extended to others themes, database or electronic libraries.
\end{abstract}

Keywords: Metric studies. Evaluation of the scientific production. Bibliometry. Evaluation of periodicals. Communication networks. 


\section{Introducción}

La evaluación del trabajo científico a través de indicadores bibliométricos es un tema que viene ganando cada vez más espacio en estudios académicos, y no es un acaso. Los diversos tipos de evaluaciones de trabajos científicos son uno de los criterios utilizados por gobiernos y órganos multinacionales, como la Unesco o la Organización Mundial de la Salud, para decidir cómo manejar sus recursos destinados a la investigación y al desarrollo.

Las evaluaciones cuantitativas a través de indicadores bibliométricos son parte de un área de estudio mayor que es el Análisis de Dominio de los trabajos científicos, utilizado internacionalmente. Esos análisis apuntan a ese o aquel científico, esa o aquella área del conocimiento, ese o aquel país, esa o aquella institución, como más fértiles o más productivos.

La propuesta de esta investigación es realizar una evaluación de la producción científica en el área de Ciencia de la Información, en el tema estudios métricos, utilizando indicadores bibliométricos. Esto es, se pretende evaluar la producción científica en el tema, utilizando como herramienta los propios estudios métricos 0 , más especialmente, los análisis bibliométricos.

\section{Objetivos}

Como objetivo general, se pretende evaluar la producción científica de periódicos pertenecientes a la base SciELO, en el área de Ciencia de la Información, en lo que se refiere al tema Estudios Métricos, a través de análisis bibliométricos.

Como objetivos específicos, se pretende identificar y analizar las tendencias presentes en la comunidad científica con relación a esa temática, en las siguientes variables: subtemas específicos abordados, palabras-clave, instituciones de origen, coautorías, autores más citados en sus referencias bibliográficas y país de origen, periódicos más citados, con la finalidad de caracterizar y subsidiar la visión actual de tendencias de investigación en ese tema, tomando como instrumento las publicaciones en el SciELO.

La elección de esa biblioteca electrónica con sus publicaciones en el formato on-line se hizo por su relevancia en el ámbito nacional e internacional y por el criterio selectivo de calidad de las revistas SciELO.

Otro factor que justifica la realización de esta investigación fue la construcción de una base de referencias actualizada en ese tema y su "esta- do del arte" en Brasil, a partir del exámen de las revistas relevantes en el área.

\section{Marco teórico}

La Scientific Electronic Library Online (SciELO) es una biblioteca electrónica que comprende una colección selecionada de periódicos científicos brasileños y resultó de un proyecto de investigación de la Fundação de Amparo à Pesquisa do Estado de São Paulo (FAPESP), en sociedad con el Centro Latino-Americano e do Caribe de Informação em Ciências da Saúde (BIREME). A partir de 2002, pasó a contar con el apoyo del Conselho Nacional de Desenvolvimento Científico e Tecnológico (CNPq).

Su objetivo es el desarrollo de una metodología común para la preparación, almacenamiento, diseminación y evaluación de la producción científica en formato electrónico. Actualmente, a través de este proyecto, son publicados 204 periódicos, incluyendo ocho campos de conocimientos: Ciencias Agrarias, Ciencias Biológicas, Ciencias de la Salud, Ciencias Exactas y de la Tierra, Ciencias Sociales y Aplicadas, Ingenierías, Lingüística, Letras y Artes. En el campo Ciencias Sociales y Aplicadas, se encuentra el área Ciencia de la Información, con publicaciones de dos revistas: Perspectivas en Ciencia de la Información y Ciencia de la Información.

El primero, Perspectivas en Ciencia de la Información, es una publicación cuatrimestral de la Escuela de Ciencia de la Información de la UFMG (Universidade Federal de Minas Gerais).

El segundo, Ciencia de la Información, incluye publicaciones cuatrimestrales de trabajos inéditos en ese área o que presenten resultados de estudios e investigaciones sobre las actividades del sector de información en ciencia y tecnología. El periódico Ciencia de la Información ha tenido fuerte actuación en el campo de la Ciencia de la Información y en el sector de información en Ciencia y Tecnología, por la vehiculación de contribuciones significativas de expertos nacionales y extranjeros, por la comprensión de su público que es constituido por la comunidad académica de investigadores y profesionales, no sólo de la Ciencia de la Información sino también de áreas próximas.

El término "Producción científica" significa la medida del volumen de libros, capítulos de libros, artículos de periódicos y otras modalidades de publicaciones impresas, digitales 0 electrónicas, que contienen resultados de investigaciones científicas de autores, instituciones, regiones, países en general o en áreas temáticas específicas. En stricto senso, la expresión 
"Producción Científica" considera la cantidad de publicaciones científicas, particularmente de artículos de periódicos, incluidos en índices bibliográficos reconocidos nacional e internacionalmente.

La producción científica, conjunto de publicaciones generadas durante la realización y término de las investigaciones, viene siendo cada vez más estudiada, especialmente en los últimos cuarenta años, cuando la explosión documental impuso, de cierto modo, la necesidad de la creación de instrumentos para evaluación de la ciencia, particularmente de instituciones de investigación e investigadores, especialmente en países como Brasil, donde el desarrollo de la ciencia está relacionado con el sistema de educación superior.

Según algunos expertos, los conceptos sobre producción científica son polémicos, cuando no divergentes. Así, según Pécora (1997, p. 159)

por producción científica se entiende toda actividad resultante de una reflexión sistemática, que implica producción original dentro de la tradición de investigación con métodos, técnicas, materiales, lenguaje propio y que contempla críticamente el patrimonio anterior de una determinada ciencia

Otros, sin embargo, militan la idea de que solo deben ser considerados producción científica los trabajos indizados en grandes bases de datos reconocidas mundialmente, entre las cuales se destaca la Web of Science, base multidisciplinaria subdividida en tres principales bases referenciales: $\mathrm{SCl}$, Science Citation Index, $\mathrm{SSCl}$-Social Science Citation Index y A\&HClArts and Humanities Citation Index.

$\mathrm{El} \mathrm{SCl}$ colecta citaciones de autores hechas en artículos publicados en las revistas que tiene indizadas (hay apenas tres brasileñas), con un total de 3.762 revistas.

Considerándose las cuestiones analizadas, se torna necesario que la producción nacional adquiera visibilidad y se vuelva más accesible. Entre las más importantes iniciativas, se cita la creación, en 1996, de la base de datos brasileña SciELO, que constituye una red de colecciones de periódicos publicados en la Internet, con acceso abierto, con la finalidad de promover la visibilidad y accesibilidad de publicaciones científicas de América Latina y del Caribe (Fundação, 2004, p. 6 e 35).

Así, los estudios de producción científica enfrentan desafíos, considerando que la producción científica es parte de un gran sistema social que es la ciencia. Según (Macias Chapula, 1998, p. 136),
[...] la ciencia necesita ser considerada como un amplio sistema social, en el cual una de sus funciones es diseminar conocimientos. Su segunda función es asegurar la preservación de estándares $y$, la tercera, es atribuir crédito y reconocimiento para aquellos cuyos trabajos han contribuido para el desarrollo de las ideas en diferentes campos.

\section{Metodología}

El procedimiento adoptado fue cuantitativo/cualitativo, en la medida en que se buscó analizar todos los datos numéricos, contextualizándolos a la luz del marco teórico adoptado y de las cuestiones emergentes en el área de Ciencia de la Información.

El procedimiento de investigación adoptado para la identificación, análisis y evaluación de los datos fue el examen de los números publicados on-line. En el primer periódico -que apenas lleva dos años de publicación on-line-, se consideró un total de 45 artículos, de los cuales solamente seis tratan de Estudios Métricos, o sea, el $13,04 \%$. En el segundo periódico, con 10 años de publicaciones on-line, fueron analizados 320 artículos, de los que sólo 39 tratan del tema en cuestión, o sea, el 12,18\%. Trabajamos así, inicialmente, con un universo de 366 artículos, siendo 45 (12,29\%, entre los dos periódicos) los artículos que tratan del tema Estudios Métricos.

A partir de la lectura de los resúmenes y, cuando fue necesario, de la lectura del texto completo, se buscó diagnosticar la producción científica, a través del examen y categorización de las subtemáticas más frecuentes producidas por los investigadores, las palabras clave presentes, las instituciones de origen más productivas relativas a los autores, las coautorías, los autores más citados en las bibliografías de esos 45 artículos, su país de origen y periódicos más relevantes.

Se trabajaron esos datos dentro de procedimientos tanto cuantitativos (bibliométricos) como cualitativos, de cara a analizarlos y contextualizarlos.

\section{Presentación de los datos y análisis de los resultados}

Inicialmente, se procedió, a partir de los dos periódicos seleccionados, a separar los artículos que tratan del tema Estudios Métricos. Se encontraron en el universo de investigación, inicialmente de 366 artículos, 45 artículos que tratan del referido tema, o sea, 12 un , 29\% del total.

Considerándose que los dos periódicos tratan de gran multiplicidad de asuntos y temas dentro 
de la Ciencia de la Información, el porcentaje de $12,29 \%$ relativo a esa especificidad en estudio muestra la relevancia del tema en el área.

A partir del conocimiento previo sobre el tema, la lectura del resumen y eventualmente la lectura del texto completo -cuando resultó necesario-, se identificaron las temáticas más frecuentes, para diagnosticar la producción científica. Los resultados se presentan en la tabla I.

Juntamente con la categorización de las subtemáticas, se asociaron las palabras clave encontradas en los diferentes artículos, buscándose siempre preservar la terminología del autor. Se observó, sin embargo, en varios momentos, que la misma palabra-clave se había utilizado para indicar asuntos diferentes, en virtud de la contigüidad temática y de vocabulario.

\begin{tabular}{|c|c|c|}
\hline Temas y palabras-clave & Frec. & $\%$ \\
\hline $\begin{array}{l}\text { Producción científica y evaluación de } \\
\text { la producción científica. (Evaluación } \\
\text { de producción científica, Producción } \\
\text { científica, Comunicación científica, } \\
\text { Producción científica brasileña, } \\
\text { Evaluación de la literatura científica, } \\
\text { Producción bibliográfica, Revistas } \\
\text { científicas) }\end{array}$ & 16 & 36 \\
\hline $\begin{array}{l}\text { Indicadores bibliométricos } \\
\text { (Indicadores bibliométricos, Ley de } \\
\text { Bradford, Indicadores, Productividad } \\
\text { de autores, number, Indicadores en } \\
\text { ciencia y tecnología, Índice de } \\
\text { dispersión, bibliometrics, Léxico } \\
\text { básico, words, Índices de citación, } \\
\text { Indicadores de innovación) }\end{array}$ & 8 & 18 \\
\hline $\begin{array}{l}\text { Informetría (Bibliometría, } \\
\text { mathematical informetrics, statistical } \\
\text { informetrics, mediametrics, } \\
\text { museometrics, webometrics, } \\
\text { Informetría Investigación operacional, } \\
\text { Caos, Técnicas informétricas }\end{array}$ & 4 & 9 \\
\hline $\begin{array}{l}\text { Redes de colaboración científica } \\
\text { (Análisis de redes sociales, redes de } \\
\text { investigación, redes de colaboración } \\
\text { científica, Redes sociales, Redes de } \\
\text { coautoría, Redes sociales, } \\
\text { Movimientos sociales, Cooperación } \\
\text { científica) }\end{array}$ & 3 & 7 \\
\hline $\begin{array}{l}\text { Cienciometría (scientometric, } \\
\text { Cienciometría) }\end{array}$ & 3 & 7 \\
\hline $\begin{array}{l}\text { Webometría (Webometría, } \\
\text { Cibermetría, Biblioteca digital, } \\
\text { Biblioteca virtual, Repositorios } \\
\text { digitales, Periódicos electrónicos) }\end{array}$ & 2 & 4 \\
\hline $\begin{array}{l}\text { Análisis de citación (Citaciones, } \\
\text { Estudio de citaciones, Análisis de } \\
\text { citación, Análisis de citación) }\end{array}$ & 2 & 4 \\
\hline $\begin{array}{l}\text { Análisis de dominio (Análisis de } \\
\text { dominio, Comunicación científica) }\end{array}$ & 2 & 4 \\
\hline
\end{tabular}

$\begin{array}{lll}\begin{array}{l}\text { Factor de impacto (Impacto } \\ \text { bibliográfico) }\end{array} & 1 & 2 \\ \text { Métodos cuantitativos (Mathematics, } & 1 & 2\end{array}$

statistics, measure, Métodos

cuantitativos de evaluación,

Metodología, Metodología para

evaluación de periódicos)

Modelaje (Información, Dispersión,

Difusión, Indicadores, Bibliometría,

Cienciometría, Informetría, Índice de

dispersión, Impacto bibliográfico)

Mensuraciones y medidas (Estudios

cuantitativos, Monitoramiento de la

información, Evaluación, Estudios de evaluación)

Información científica (Información

científica, documents, Indización;

Recuperación de la información,

Acceso a la información científica,

Comunicación científica)

\begin{tabular}{lll}
\hline TOTAL & 45 & 100
\end{tabular}

Tabla I. Temas encontrados, palabras-clave y frecuencias

Los temas más frecuentes son la producción científica y las cuestiones relacionadas con la evaluación de revistas, evaluación de la producción científica, indicadores métricos - tales como factor de impacto- y análisis de citación, totalizando el $48 \%$ de artículos. Los artículos que trabajan con webometría, links, evaluaciones informétricas, cientométricas y bibliométricas también han sido incluidos, así como métodos cuantitativos en general, estos en menor porcentual. Observamos que artículos que trabajan con cuestiones más candentes en el tema, tales como análisis de dominio y redes de comunicación científica, son contemplados, aunque con baja frecuencia.

Otra variable en estudio se refirió a las instituciones de origen de los artículos, con la finalidad de averiguar el origen institucional de los investigadores que se preocupan con esas cuestiones, en el sentido de tabular los focos de producción. Los resultados están presentados en la tabla II. Analizando los datos de la tabla II, se concluye que: aproximadamente, el $18 \%$ de los artículos son producciones de otros países, evidenciando el carácter internacional de la biblioteca electrónica en estudio; el $20 \%$ de los artículos fueron producidos por la Universidade de São Paulo y su sistema de bibliotecas; la casi totalidad del restante, el $55 \%$, fueron producidos por las diferentes universidades del país, demostrando que es prioritariamente en la universidad donde se produce investigación, a pesar de la multiplicidad de institutos de investigación existentes. Solamente el $7 \%$ de las publicaciones vienen de institutos de investigación. 


\begin{tabular}{|c|c|c|}
\hline $\begin{array}{l}\text { Instituciones de origen de los } \\
\text { investigadores }\end{array}$ & Frec. & $\%$ \\
\hline $\begin{array}{l}\text { Universidade de São Paulo (USP) y su } \\
\text { sistema de bibliotecas }\end{array}$ & 9 & 20 \\
\hline $\begin{array}{l}\text { Producción internacional (México, } \\
\text { Paraguay, Bélgica, España, EE. UU. y } \\
\text { Francia) }\end{array}$ & 8 & 18 \\
\hline $\begin{array}{l}\text { Universidade Federal de Minas Gerais } \\
\text { (UFMG) }\end{array}$ & 5 & 11 \\
\hline $\begin{array}{l}\text { Universidade Estadual y Federal de } \\
\text { Santa Catarina (UDESC y UFSC) }\end{array}$ & 4 & 9 \\
\hline Universidade Federal da Bahia (UFBA) & 4 & 9 \\
\hline $\begin{array}{l}\text { Universidade Federal do Rio Grande } \\
\text { do Sul (UFRGS) }\end{array}$ & 3 & 7 \\
\hline Universidade de Brasília (UNB) & 3 & 7 \\
\hline $\begin{array}{l}\text { Universidade Federal do Paraná } \\
\text { (UFPR) }\end{array}$ & 2 & 4 \\
\hline $\begin{array}{l}\text { Universidades Federais do Espírito } \\
\text { Santo, Paraíba, Piauí y Maranhão } \\
\text { (UFES), (UFPI), (UFPA) y (UFMA) }\end{array}$ & 4 & 9 \\
\hline $\begin{array}{l}\text { Instituciones diversas (BIREME, IBICT } \\
\text { y EMBRAPA) }\end{array}$ & 3 & 7 \\
\hline Total & 45 & 100 \\
\hline
\end{tabular}

Tabla II. Instituciones de origen de los investigadores estudiados

La coautoría es otra de las variables estudiadas, con el objetivo de diagnosticar los grupos de investigación, líneas de investigación y colegios invisibles que estudian el tema, para tabular posibles redes de comunicación. Sin embargo, en virtud del universo un tanto restricto, no se encontraron grupos de investigadores que publicaran conjuntamente. Dicho sea esto sin considerar el estudio de las cocitaciones, que supera el alcance de este trabajo.

\begin{tabular}{l|ll}
\hline Número de autores & Frecuencia & $\%$ \\
\hline Un autor & 22 artículos & 48,8 \\
Dos autores & 14 artículos & 31,1 \\
Tres autores o más & 9 artículos & 20,0 \\
\hline Total & 45 & 100 \\
\hline
\end{tabular}

Tabla III. Coautorías (número de autores por artículo)

Así, el $51,1 \%$ de los artículos son elaborados a partir de coautorías de dos o más autores. Esa tendencia es mundial, ya que los trabajos en coautorías han sido incentivados por las instituciones de fomento de la investigación.

En cuanto a la frecuencia de autoría, se observa que dos únicos autores publicaron dos artículos en la revista Ciencia de la Información en ese periodo de tiempo estudiado: Nádia Vanti y Daisy Pires Noronha, esta última con un trabajo en sociedad con Dinah Aguiar Poblacion. Como será visto posteriormente, estas dos investiga- doras obtuvieron número representativo de citaciones en los artículos publicados.

\begin{tabular}{|c|c|}
\hline Autores & $N^{\circ}$ \\
\hline Rousseau, R. (Bélgica) & 17 \\
\hline Spinak, Ernesto (Venezuela) & 15 \\
\hline Price, Derek Solla (EE. UU.) & 13 \\
\hline Newmam, M.E.J. (EE. UU.) & 10 \\
\hline Courtial, Jean Paul (EE. UU.) & 10 \\
\hline Braga, Gilda Maria (Brasil) & 9 \\
\hline Glanzel, Wolfgang (Alemania) & 9 \\
\hline Robredo, Jaime (Brasil) & 9 \\
\hline Velho, Léa (Brasil) & 9 \\
\hline Beaver, Donald B. (EE. UU.) & 8 \\
\hline Castro, Cláudio Moura (Brasil) & 8 \\
\hline Callon, Michel (España) & 7 \\
\hline Meadows, A. J. (Inglaterra) & 7 \\
\hline Vinkler, P. (Hungría) & 7 \\
\hline Bourdieu, Pierre (Francia) & 6 \\
\hline Institute for Scientific Information (EE. UU.) & 6 \\
\hline Katz, J. Sylvan (Inglaterra) & 6 \\
\hline Trzesniak, Piotr. (Brasil) & 6 \\
\hline Vanti, Nadia A. P. (Brasil) & 6 \\
\hline Población, Dinah Aguiar (Brasil) & 6 \\
\hline Ravichandra, Rao (India) & 6 \\
\hline Borgatti, S. P. (EE. UU.) & 5 \\
\hline Bufrem, Leilah Santiago (Brasil) & 5 \\
\hline Castro, Regina Célia Figueiredo (Brasil) & 5 \\
\hline Meneghini, Rogério (Brasil) & 5 \\
\hline Moed, H.F. (Holanda) & 5 \\
\hline Moya-Anegón, Félix (España) & 5 \\
\hline Oberhofer, Cecília Alves (Brasil) & 5 \\
\hline Polanco, X. (Francia) & 5 \\
\hline Sengupta, I. N (India) & 5 \\
\hline White, Howard D. (EE. UU.) & 5 \\
\hline Brookes, B. C. (Inglaterra) & 4 \\
\hline Crane, Diana (EE. UU.) & 4 \\
\hline Garfield, Eugene (EE. UU.) & 4 \\
\hline Mcgrath, W. E (Canadá) & 4 \\
\hline Moravcsik, M.J. (EE. UU.) & 4 \\
\hline Mueller, Suzana Pinheiro Machado (Brasil) & 4 \\
\hline Narin, Francis (Luxemburgo) & 4 \\
\hline Noronha, Daisy Pires (Brasil) & 4 \\
\hline Oliveira, Marlene De (Brasil) & 4 \\
\hline Pinheiro, Lena Vania Ribeiro (Brasil) & 4 \\
\hline Romão, W. (Brasil) & 4 \\
\hline Tague-Sutcliffe, J. (Brasil) & 4 \\
\hline Targino, Maria Das Graças (Brasil) & 4 \\
\hline Testa, James (Brasil) & 4 \\
\hline Watts, Duncan (EE. UU.) & 4 \\
\hline Whitlow, Edith (EE. UU.) & 4 \\
\hline
\end{tabular}

Tabla IV: Autores citados, procedencia y número de citaciones 
Otra variable estudiada en esta investigación fue el análisis de las citaciones de los diferentes 45 autores, a fin de averiguar en cuales fuentes de información los investigadores buscan sus referencias, pudiéndose tabular, a través de ella, un posible frente de investigación en la temática.

Fueron examinadas, del total de 45 artículos, aproximadamente 765 citaciones, con una media de 17,1 citaciones por artículo.

Se tabularon solo los autores que fueron citados 4 o más veces, considerándose aquí las coautorías y las autorías individuales, que también fueron contadas; lo que resultó en un un ranking de 47 investigadores que, ordenados de modo decreciente por número de citaciones hechas, se presentan en la tabla IV, en la página anterior.

Se consideró pertinente registrar el país de origen de esos investigadores, para localizar los países más productivos. EE. UU. aparece como uno de los países más citados $(24 \%$, en un universo de 294 citaciones), probablemente por ser uno de los primeros países que iniciaron los estudios en este área, perpetuando su posición de grandes productores en esos estudios, hasta los días de hoy.

Aunque según un estudio recién realizado por el Centro Latino-Americano e do Caribe de Informação em Ciências da Saúde (BIREME) con apoyo de la Fundação de Amparo à Pesquisa do Estado de São Paulo (FAPESP) y del Conselho Nacional de Pesquisa (CNPq), (2008), todavía pendiente de ser publicado, la investigación sobre el tema se ha incrementado en los últimos 30 años; es importante observar que, en Brasil, en los últimos 20 años, los autores brasileños crecieron tres veces más que el total mundial, hecho comprobado por ese examen, pues el $15 \%$ de las citaciones son para investigadores brasileños, porcentaje considerado significativo. Sin embargo, no se puede dejar de registrar el gran número de citaciones de autores extranjeros, alrededor del 85\%, advenidos también de otros países, en los que las cuestiones relativas a la producción científica, evaluación de la ciencia y métricas en general constituyen aspectos permanentemente apoyados por las políticas científicas.

Con relación al número de citaciones, los más citados son investigadores extranjeros: en primer lugar, Ronald Rousseau de Bélgica, con 17 citaciones; después, Ernesto Spinak, de Venezuela, con 13 citaciones; Solla Price, de EE. UU., con 13 citaciones. Brasil aparece en sexto lugar con la investigadora brasileña Gilda Maria Braga, de Rio de Janeiro, una de las pioneras en esos estudios, así como Jaime Robredo, de la Universidade de Brasília, y Léa Velho, todos con 9 citaciones.

Se observa gran dispersión de las citaciones, pues, de las 765, sólo 47 fueron contempladas 4 o más veces, considerando también las autorías múltiples. Los restantes autores aparecen 2 ó 3 veces y la gran mayoría es citada sólo una vez, lo que sugiere que hay muchos investigadores produciendo en el área, pero con baja productividad y poco reconocidos.

Hay que destacar también el carácter multidisciplinario del tema, hecho que causa una pulverización grande de autores procedentes de las más diferentes áreas de conocimiento, que pasaron a preocuparse en los últimos años con cuestiones cienciométricas.

Se buscó evaluar también, en los artículos citados por los autores de las revistas en estudio, en cuáles revistas fueron publicados. Se averiguó que 46 de ellos fueron publicados en la revista Ciencia de la Información -lo que muestra la gran penetración de la revista en el área-, y 39 de ellos fueron publicados en la revista Scientometrics, que, según estudios ya realizados, es "lo más completo en artículos dirigidos a los Estudios Métricos" (Pinto et al., 2007)

Los demás fueron publicados en otras revistas, concretamente Journal Management, Journal Information Science, Informetrics, Journal of Documentation, Proocedings of Nacional Academy Sciences, Comunicación científica (revista nacional), Information Processures \& Manegement, y Journal of the American Society for Information Science and techonology, entre otras.

\section{Conclusiones y recomendaciones}

La investigación realizada tuvo como propósito analizar la producción científica nacional sobre estudios métricos más visible y accesible por medio electrónico a través del SciELO, y, al mismo tiempo, crear una base de datos a través de la cual sea posible evaluar la producción científica del país y aumentar su visibilidad internacional. Además, la base SciELO se demuestra como una plataforma clave para estudiar la producción nacional en el área, con vistas al establecimiento de nuevas acciones de política científica.

Con relación a los resultados, aunque se haya trabajado con sólo 45 artículos, éstos retratan adecuadamente las características de esos estudios en Brasil: las cuestiones relativas a la evaluación de la producción científica son las 
más relevantes en el tema; la cuestión de coautoría sigue la tendencia mundial de producción en sociedades y grupos; los autores más citados por los 45 artículos son bien reconocidos dentro del tema Estudios Métricos, en el ámbito nacional e internacionalmente por su productividad de excelencia; y las revistas más citadas por esos 45 autores son, en el ámbito nacional, la revista Ciencia de la Información y, en ámbito internacional, la revista Scientometrics.

Como recomendación, se sugiere que estudios de esa naturaleza sean realizados en diferentes áreas de estudios y en diferentes fuentes de Información, de modo todavía más amplio, para hacer la ciencia más visible en sus diferentes campos.

\section{Referencias}

Anegón, Félix de Moya. Solana, Vítor Herrera (2000). Visibilidad internacional de la producción científica Iberoamericana en Biblioteconomía Y Documentación (1991-1999). // V Encuentro de EDIBICIC, Granada, España, 2000. 341-368.

Anegón, Félix de Moya; Rodrigues, Zaída Chinchilla; Alvarez, Elena Corera; Quesada, Benjamim Vargas; Fernandez, Francisco Muñoz; Solana, Victor Herrera (2005). Análisis de Dominio institucional: La Producción Científica de la Universidad de Granada (SCl 1991-99). // Revista Espanõla de la Documentación Científica. 28:2 (2005). 170-195

Bufrem, Leilah Santiago et al. (2003). Organização do conhecimento: tendências da produção científica. // Frias, José Antonio; Traveso Críspulo (Org). Tendencia de la investigación en organización del conocimiento. Salamanca: Ediciones Universidad Salamanca, 2003. 321326.

Funaro, Vania M.B.O.; Noronha, Daysi Pires. Literatura cinzenta: canais de distribuição e incidência de bases de dados (2006). // Población, Dinah Aguiar; Witter, Geraldina Porto; Silva, José Fernando Modesto da (orgs.). Comunicação e produção científica: contexto, indicadores e avaliação, São Paulo: Angelara, 2006, p. 215-234.

Fundação de amparo à pesquisa do estado de São Paulo FAPESP (2005). Análise da produção científica a partir de indicadores bibliométricos. // Indicadores de ciência, tecnologia e inovação em São Paulo. 1. São Paulo: Editora FAPESP, 2005

Macias-Chapula, C. A (1998). O papel da informetria e da cienciometria e sua perspectiva nacional e internacional. // Ciência da Informação. 27:2 (1998). http://www.ibict. br/cionline/ em 04 de abril de 2008.

Meneghini, Rogério (1998). Avaliação da produção científica e o projeto SciELO // Ciência da Informação. 27:2. (1998).

Meneghini, Rogério; Packer, Abel L. (2008). A Amplitude da autoria Multidisciplinar de artigos sobre Cienciometria no Brasil. // BIREME, OPAS-OMS, fevereiro, 2008 (no prelo).

Pécora, Gláucia Maria Mollo (1997). Atividades acadêmicas de pesquisador. // Witter, Geraldina Porto (org). Produção científica: Átomo, 1997. 157-167.

Pinto, A. L. et al. (2007). Indicadores científicos na literatura em Bibliometria e Cientometria, através da redes sociais
// Brazilian Journal of Information Science. 1:1 (2007) http://www.bjis.unesp. br em 25 de maio de 2008.

Silva, J. F. M. (2006). Comunicação e produção científica: contexto, indicadores e avaliação. São Paulo: Angelara, 2006.

Spinak, E. Dicionário enciclopédico de bibliometria, cienciometria e informetria. Caracas: Unesco - CII/II, 1996.

Strehl, Letícia. O fator de impacto do ISI e a avaliação da produção científica: aspectos conceituais e metodológicos (2005) // Ciência da Informação. 34:1 (2005). http:// www.scielo.br/scielo.php?script=sci_arttext\&pid=\$0100 $19652005000100003 \&$ Ing=en\&nrm=isso (22 de abril de 2008).

Velho, Lea M.L.S (2004). Cuidado com os rankings cientificos. http://www.prometeu.com.br/bb-lea.asp (22 de agosto de 2004). 